Journal of Advanced Research in Fluid Mechanics and Thermal Sciences

Journal homepage: www.akademiabaru.com/arfmts.html ISSN: 2289-7879

\title{
Unsteady Free Convection Flow of Nanofluids between Vertical Oscillating Plates with Mass Diffusion
}

\author{
Fasihah Zulkiflee ${ }^{1}$, Sharidan Shafie ${ }^{1,{ }^{*}}$, Ahmad Qushairi Mohamad $^{1}$ \\ Department of Mathematical Sciences, Fakulti Sains, Universiti Teknologi Malaysia, 81310 Skudai, Johor, Malaysia
}

\begin{tabular}{ll}
\hline ARTICLE INFO & ABSTRACT \\
\hline $\begin{array}{l}\text { Article history: } \\
\text { Received 29 May 2020 }\end{array}$ & $\begin{array}{l}\text { In this paper, free convection of nanofluids flow with oscillating vertical parallel plates } \\
\text { and mass diffusion were considered. Obtained equations were converted into ordinary }\end{array}$ \\
$\begin{array}{l}\text { Accepted } 9 \text { September } 2020 \\
\text { Available online } 18 \text { October } 2020\end{array}$ & $\begin{array}{l}\text { differential equations with appropriate transformations. Method of Laplace transform } \\
\text { was used to find the exact solutions of velocity, temperature and concentration } \\
\text { profiles from the dimensionless governing equations. Discussion of graph pertaining to } \\
\text { different embedded parameters such as Prandtl number, Schmidt number, Grashof } \\
\text { and mass Grashof number, oscillating parameter and nanoparticles volume fraction } \\
\text { parameter was also added. Skin friction, Nusselt and Sherwood number were also } \\
\text { discussed and deliberated. }\end{array}$
\end{tabular}

Keywords:

Nanofluids; Parallel Plates; Oscillating;

Mass Diffusion

Copyright $@ 2020$ PENERBIT AKADEMIA BARU - All rights reserved

\section{Introduction}

The study of free convection flows between two parallel plates has attracted much attention due to the various applications in engineering areas including the purification of crude oil, pumps accelerators, petroleum industry and power generators. In particular, researchers have investigated free convection flows with different parameter and boundary conditions such as magnetohydrodynamic (MHD), porous medium, radiation, chemical reaction, constant wall temperature and heat flux, viscous dissipation and oscillating problem. Singh et al., [1] investigated free convection flow between two vertical parallel plates and the results shows that it is in good agreement with the steady state problem solutions. Marneni [2] investigated the effects of thermal radiation on the unsteady Couette flow between two parallel plates with constant heat flux at one boundary and solved the problem using Laplace transform. Meanwhile, Rajput and Sahu [3] considered constant temperature and variable mass diffusion in their investigation and determine that the velocity and skin friction of the fluid decreased the value of Prandtl number, Schmidt number and Magnetic parameter. Vidhya and Kesavan [4] investigated laminar convection through porous

\footnotetext{
${ }^{*}$ Corresponding author.

E-mail address: sharidan@utm.my

https://doi.org/10.37934/arfmts.76.2.118131
} 
medium between two vertical parallel plates with heat source. Umavathi and Shekar [5] studied the combined effect of variable viscosity and thermal conductivity on free convection flow of a viscous fluid in a vertical channel using DTM. The study of oscillatory flow through a channel is important in science and engineering applications such as in MHD generators, blood flow and in petroleum engineering [6]. Some researchers considered oscillation in their research in vertical plates but not many considered oscillating in parallel plates [7-9]. The studies show that analysis in oscillating flow in channel are only a few. Chen et al., [10] studied heat transfer characteristics of oscillating flow regenerator filled with circular tubes or parallel plates. The results show that the mean temperature gradient gives greatest contribution to the heat transfer performance of oscillating flow. Umavathi et al., [11] investigated unsteady oscillatory flow and heat transfer in a horizontal composite porous medium channel. Meanwhile, MHD oscillatory free convection flow past parallel plates with periodic temperature and concentration was investigated by Sharma and Dutt [12]. Das et al., [13] investigated oscillatory MHD convective flow in a vertical channel filled with porous medium with Hall and thermal radiation effects. Falade et al., [6] studied MHD oscillatory flow through a porous channel saturated with porous medium. It was found that skin friction increases on both channel plates as injection increases on the heated plate.

A nanofluid is a fluid containing nanometre-sized particles that are called nanoparticles. These fluids are engineered colloidal suspensions of nanoparticles in a base fluid. The nanoparticles used in nanofluids are typically made of oxides, carbides, metals, or carbon nanotubes. According to Choi et al., [14], fluid is enhanced when a small number of nanoparticles is added in the fluid. Due to the enhanced heat transfer characteristics, it becomes important in science and engineering and some of the applications in science and engineering include cooling technologies such as cooling microchips in automotive, nuclear reactors as well as in biomedicine [15]. Narahari et al., [16] investigated the exact solutions of the free convection flow of nanofluids between two vertical parallel plates with radiation effects. Hajizadeh et al., [17] discussed free convection flow of nanofluids between vertical plates with damped thermal flux boundary condition. Shekolaleslami and Ganji [18] and Shekolaleslami et al., [19] studied heat transfer of Cu-water nanofluid flow between parallel plates and extended the study and investigated the numerical simulation of unsteady nanofluid flow and heat transfer between parallel plates with presence of a time-dependent magnetic field. Dogonchi et al., [20] studied flow and heat transfer of MHD nanofluid between parallel plates with thermal radiation effect.

Motivated by the above investigations, since there are only a few researches on oscillations between two parallel plates, we would like to consider investigating oscillating nanofluid. The present work deals with oscillatory free convection of nanofluid flow between two parallel plates with mass diffusion. Water-Cu nanofluid was considered in this study. This problem will be solved using Laplace transform method and the solutions are presented in graphs.

\section{Mathematical Formulation}

Consider an unsteady free convection nanofluid flow between two parallel plates with mass diffusion. An oscillating plate at $y=0$ was also considered in this study. The $x^{\prime}$-axis was considered along one of the vertical plates and the $y^{\prime}$-axis was taken as normal to the plates. Figure 1 shows the flow configuration of the flow and the governing equations under the normal Boussinesq' approximation as follows. 


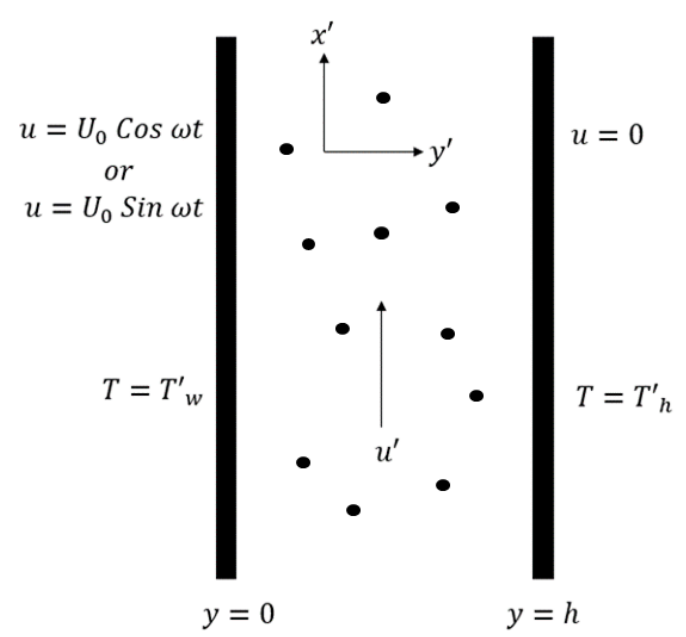

Fig. 1. Flow configuration of the problem

$\rho_{n f} \frac{\partial u \prime}{\partial t^{\prime}}=\mu_{n f} \frac{\partial^{2} u \prime}{\partial y^{\prime 2}}+g\left(\rho \beta_{T}\right)_{n f}\left(T^{\prime}-T_{h}^{\prime}\right)+g\left(\rho \beta_{C}\right)_{n f}\left(C^{\prime}-C_{h}^{\prime}\right)$

$\left(\rho C_{p}\right)_{n f} \frac{\partial T^{\prime}}{\partial t^{\prime}}=k_{n f} \frac{\partial^{2} T^{\prime}}{\partial y^{\prime 2}}$

$\frac{\partial C^{\prime}}{\partial t^{\prime}}=D_{n f} \frac{\partial^{2} C^{\prime}}{\partial y^{\prime 2}}$

subject to initial and boundary conditions

$t \leq 0: u^{\prime}=0, T^{\prime}=T_{h}^{\prime}, C^{\prime}=C_{h}^{\prime}$ for $0 \leq y^{\prime} \leq h$

$t>0: u^{\prime}=U_{0} \cos \omega t$ and $U_{0} \sin \omega t, T^{\prime}=T_{w}^{\prime}, C^{\prime}=C_{w}^{\prime}$ at $y^{\prime}=0$

$u^{\prime}=0, T^{\prime}=T_{h}^{\prime}, C^{\prime}=C_{h}^{\prime}$ at $y^{\prime}=h$

Here $u^{\prime}$ is the velocity of the fluid, $g$ is the acceleration due to gravity, $\left(\rho \beta_{T}\right)_{n f}$ is the volumetric coefficient of thermal expansion of nanofluid, while $\left(\rho \beta_{C}\right)_{n f}$ is the volumetric coefficient of concentration expansion, $t^{\prime}$ is time, $h$ is the distance between two plates, $T^{\prime}$ is the temperature of the fluid, $T_{h}^{\prime}$ is the temperature of the plate at $y^{\prime}=h, C^{\prime}$ is the concentration of the fluid while $C_{h}^{\prime}$ is the concentration of the plate at $y^{\prime}=h, \rho_{n f}$ is the density of the fluid, $\left(C_{p}\right)_{n f}$ is specific heat at constant pressure, $D_{n f}$ is mass diffusion coefficient, $v_{n f}$ is the kinematic viscosity of nanofluid, $k_{n f}$ is the thermal conductivity of nanofluid and $C_{w}^{\prime}$ concentration of the fluid at $y^{\prime}=0$. For the nanofluids expressions for $\mu_{n f}, \rho_{n f},\left(\rho C_{p}\right)_{n f},\left(\rho \beta_{T}\right)_{n f}$ and $\left(\rho \beta_{C}\right)_{n f}$ are given by Pal et al., [21]

$$
\begin{aligned}
& \mu_{n f}=\frac{\mu_{f}}{(1-\phi)^{2.5}} \\
& \rho_{n f}=(1-\phi) \rho_{f}+\phi \rho_{s} \\
& \left(\rho C_{p}\right)_{n f}=(1-\phi)\left(\rho C_{p}\right)_{f}+\phi\left(\rho C_{p}\right)_{s} \\
& \left(\rho \beta_{T}\right)_{n f}=(1-\phi)\left(\rho \beta_{T}\right)_{f}+\phi\left(\rho \beta_{T}\right)_{S} \\
& \left(\rho \beta_{C}\right)_{n f}=(1-\phi)\left(\rho \beta_{C}\right)_{f}+\phi\left(\rho \beta_{C}\right)_{s}
\end{aligned}
$$


where $\phi$ is the nanoparticle volume fraction, $\rho_{f}$ is the density of the base fluids, $\rho_{s}$ is the density of solid particle, $C_{p}$ is the specific heat at constant pressure. Table 1 shows the thermal conductivity of the nanofluid is given by Pal et al., [21] and Feng and Johnson [22]

$$
\begin{aligned}
& k_{n f}=\frac{k_{f}\left(k_{S}+2 k_{f}\right)-2 \phi\left(k_{f}-k_{S}\right)}{\left(k_{S}+2 k_{f}\right)+\phi\left(k_{f}-k_{S}\right)} \\
& D_{n f}=\frac{(1-\phi)}{1+\frac{\phi}{2}} D_{f}
\end{aligned}
$$

Table 1

Thermal conductivity of nanofluid

\begin{tabular}{lllll}
\hline & Water & Kerosene & $\mathrm{Cu}$ & $\mathrm{Ag}$ \\
\hline$\rho\left(\frac{\mathrm{kg}}{\mathrm{m}^{3}}\right)$ & 997.1 & 780 & 8933 & 10500 \\
$C_{p}\left(\frac{J}{\mathrm{kgK}}\right)$ & 4179 & 2090 & 385 & 235 \\
$\rho\left(\frac{W}{m K}\right)$ & 0.613 & 0.149 & 401 & 429 \\
$\beta\left(K^{-1}\right)$ & 0.00021 & 0.00099 & 0.0000167 & 0.0000189 \\
\hline
\end{tabular}

Cu-water nanofluids are considered in this research because they have high thermal conductivity, make good compatibility with basic liquid and the cost is not too high. The dimensionless variables are introduced

$y=\frac{y^{\prime}}{U_{0}}, \quad t=\frac{t^{\prime} v_{f}}{U_{0}^{2}}, u=\frac{u^{\prime} U_{0}}{v_{f}}$,

$\theta=\frac{T^{\prime}-T_{h}^{\prime}}{T_{w}^{\prime}-T_{h}^{\prime}}, \mu_{f}=\rho_{f} v_{f}, C=\frac{C^{\prime}-C_{h}^{\prime}}{C_{w}^{\prime}-C_{h}^{\prime}}$

Eq. (1)-(4) by using Eq. (5)-(7) will be reduced to following dimensionless equations

$\frac{\partial u}{\partial t}=a_{1} \frac{\partial^{2} u}{\partial y^{2}}+a_{2} G r \theta+a_{3} G m C$

$a_{4} \operatorname{Pr} \frac{\partial \theta}{\partial t}=\frac{\partial^{2} \theta}{\partial y^{2}}$

$\frac{\partial C}{\partial t}=\frac{a_{5}}{S c} \frac{\partial^{2} C}{\partial y^{2}}$

and the initial and boundary conditions are

$t \leq 0: u=0, \theta=0, C=0$ for $0 \leq y \leq 1$

$t>0: u=\cos \omega t$ and $\sin \omega t, \theta=1, C=1$ at $y=0$

$u=0, \theta=0, C=0$ at $y=1$ 
where $S c=\frac{v_{f}}{D_{f}}$ is the Shmidt number, $G r=\frac{g U_{0}^{3}\left(\beta_{T}\right)_{f}\left(T_{w}^{\prime}-T_{h}^{\prime}\right)}{\left(v_{f}\right)^{2}}$ is the thermal Grashof number, $G m=$ $\frac{g U_{0}^{3}\left(\beta_{C}\right)_{f}\left(C_{w}^{\prime}-C_{h}^{\prime}\right)}{\left(v_{f}\right)^{2}}$ is mass Grashof number, $\operatorname{Pr}=\frac{\left(\rho C_{p}\right)_{f} v_{f}}{k_{f}}$ is Prandtl number and $\omega t$ is the oscillating parameter. Eq. (8)-(10) with boundary condition Eq. (11) would be solved by using Laplace transform.

\section{Solutions of the Problem}

The solutions of velocity profile, temperature profile and concentration profile after solving the Eq. (6), Eq. (7) and Eq. (8) with the initial and boundary conditions Eq. (9) by using Laplace transform method are obtained as

$$
\begin{aligned}
& U_{c}(y, q)=\sum_{n=0}^{\infty}\left[\frac{\beta_{3}}{q^{2}} e^{-a \sqrt{\frac{q}{a_{1}}}}-\frac{\beta_{3}}{q^{2}} e^{-b \sqrt{\frac{q}{a_{1}}}}+\frac{1}{2(q-i \omega)} e^{-a \sqrt{\frac{q}{a_{1}}}}-\frac{1}{2(q-i \omega)} e^{-b \sqrt{\frac{q}{a_{1}}}}+\frac{1}{2(q+i \omega)} e^{-a \sqrt{\frac{q}{a_{1}}}}-\right. \\
& \left.\frac{1}{2(q+i \omega)} e^{-b \sqrt{\frac{q}{a_{1}}}}-\frac{\beta_{1}}{q^{2}} e^{-a \sqrt{a_{4} \operatorname{Pr} q}}+\frac{\beta_{1}}{q^{2}} e^{-b \sqrt{a_{4} \operatorname{Pr} q}}-\frac{\beta_{2}}{q^{2}} e^{-a \sqrt{\frac{S c q}{a_{5}}}}+\frac{\beta_{2}}{q^{2}} e^{-b \sqrt{\frac{S c q}{a_{5}}}}\right] \\
& U_{S}(y, q)=\sum_{n=0}^{\infty}\left[\frac{\beta_{3}}{q^{2}} e^{-a \sqrt{\frac{q}{a_{1}}}}-\frac{\beta_{3}}{q^{2}} e^{-b \sqrt{\frac{q}{a_{1}}}}+\frac{1}{2(q-i \omega)} e^{-a \sqrt{\frac{q}{a_{1}}}}-\frac{1}{2(q-i \omega)} e^{-b \sqrt{\frac{q}{a_{1}}}}-\frac{1}{2(q+i \omega)} e^{-a \sqrt{\frac{q}{a_{1}}}}+\right. \\
& \left.\frac{1}{2(q+i \omega)} e^{-b \sqrt{\frac{q}{a_{1}}}}-\frac{\beta_{1}}{q^{2}} e^{-a \sqrt{a_{4} \operatorname{Pr} q}}+\frac{\beta_{1}}{q^{2}} e^{-b \sqrt{a_{4} \operatorname{Pr} q}}-\frac{\beta_{2}}{q^{2}} e^{-a \sqrt{\frac{S c q}{a_{5}}}}+\frac{\beta_{2}}{q^{2}} e^{-b \sqrt{\frac{S c q}{a_{5}}}}\right] \\
& \bar{\theta}(y, q)=\sum_{n=0}^{\infty}\left[\frac{1}{q} e^{-a \sqrt{a_{4} \operatorname{Pr} q}}-\frac{1}{q} e^{-b \sqrt{a_{4} \operatorname{Pr} q}}\right] \\
& \bar{C}(y, q)=\sum_{n=0}^{\infty}\left[\frac{1}{q} e^{-a \sqrt{\frac{S c q}{a_{5}}}}-\frac{1}{q} e^{-b \sqrt{\frac{S c q}{a_{5}}}}\right]
\end{aligned}
$$

Eq. (18)-(20) were transformed using inverse Laplace and the solution in term $(y, t)$ are as follows

$$
\begin{aligned}
& u_{c}(y, t)=\sum_{n=0}^{\infty}\left[\beta_{3}\left(f_{1}\left(\frac{a}{\sqrt{a_{1}}}, t\right)-f_{1}\left(\frac{b}{\sqrt{a_{1}}}, t\right)\right)+f_{2}\left(\frac{a}{\sqrt{a_{1}}}, t\right)-f_{2}\left(\frac{b}{\sqrt{a_{1}}}, t\right)+f_{3}\left(\frac{a}{\sqrt{a_{1}}}, t\right)-\right. \\
& \left.f_{3}\left(\frac{b}{\sqrt{a_{1}}}, t\right)+\beta_{3}\left(-f_{1}\left(a \sqrt{a_{4} P r}, t\right)+f_{1}\left(b \sqrt{a_{4} P r}, t\right)\right)+\beta_{2}\left(-f_{1}\left(a \sqrt{\frac{S c}{a_{5}}}, t\right)+f_{1}\left(b \sqrt{\frac{S c}{a_{5}}}, t\right)\right)\right] \\
& u_{s}(y, t)=\sum_{n=0}^{\infty}\left[\beta_{3}\left(f_{1}\left(\frac{a}{\sqrt{a_{1}}}, t\right)-f_{1}\left(\frac{b}{\sqrt{a_{1}}}, t\right)\right)+f_{2}\left(\frac{a}{\sqrt{a_{1}}}, t\right)-f_{2}\left(\frac{b}{\sqrt{a_{1}}}, t\right)-f_{3}\left(\frac{a}{\sqrt{a_{1}}}, t\right)+\right. \\
& \left.f_{3}\left(\frac{b}{\sqrt{a_{1}}}, t\right)+\beta_{3}\left(-f_{1}\left(a \sqrt{a_{4} P r}, t\right)+f_{1}\left(b \sqrt{a_{4} P r}, t\right)\right)+\beta_{2}\left(-f_{1}\left(a \sqrt{\frac{S c}{a_{5}}}, t\right)+f_{1}\left(b \sqrt{\frac{S c}{a_{5}}}, t\right)\right)\right] \\
& \theta(y, t)=\sum_{n=0}^{\infty}\left[\operatorname{Erfc}\left(\frac{a \sqrt{a_{4} P r}}{2 \sqrt{t}}\right)-\operatorname{Erfc}\left(\frac{b \sqrt{a_{4} P r}}{2 \sqrt{t}}\right)\right] \\
& C(y, t)=\sum_{n=0}^{\infty}\left[\operatorname{Erfc}\left(\frac{a \sqrt{S c}}{2 \sqrt{a_{5} t}}\right)-\operatorname{Erfc}\left(\frac{b \sqrt{S c}}{2 \sqrt{a_{5} t}}\right)\right]
\end{aligned}
$$


where,

$a=2 n+y, \quad b=2 n+2-y$

$a_{1}=\frac{1}{(1-\phi)^{2.5}\left((1-\phi)+\phi \frac{\rho_{s}}{\rho_{f}}\right)}$

$a_{2}=\frac{1-\phi+\phi \frac{\left(\rho \beta_{T}\right)_{s}}{\left(\rho \beta_{T}\right)_{f}}}{1-\phi+\phi \frac{\rho_{S}}{\rho_{f}}}, \quad a_{3}=\frac{1-\phi+\phi \frac{\left(\rho \beta_{C}\right)_{s}}{\left(\rho \beta_{C}\right)_{f}}}{1-\phi+\phi \frac{\rho_{S}}{\rho_{f}}}$

$a_{4}=\left(1-\phi+\phi \frac{\left(\rho C_{p}\right)_{s}}{\left(\rho C_{p}\right)_{f}}\right)\left(\frac{k_{S}+2 k_{f}+\phi\left(k_{f}-k_{S}\right)}{k_{S}+2 k_{f}-2 \phi\left(k_{f}-k_{S}\right)}\right), \quad a_{5}=\frac{1-\phi}{1+\frac{\phi}{2}}$

$\beta_{1}=\frac{a_{2} G r}{a_{1}\left(a_{4} P r-1\right)}, \quad \beta_{2}=\frac{a_{3} G m}{a_{1}\left(\frac{s c}{a_{5}}-1\right)}, \quad \beta_{3}=\beta_{1}+\beta_{2}$

$f_{1}(z, t)=\left(\frac{z^{2}}{2}+t\right) \operatorname{Erfc}\left(\frac{z}{2 \sqrt{t}}\right)-z \sqrt{\frac{t}{\pi}} e^{-\frac{z^{2}}{4 t}}$

$f_{2}(z, t)=\frac{e^{i \omega t}}{4}\left(e^{z \sqrt{i \omega}} \operatorname{Erfc}\left(\frac{z}{2 \sqrt{t}}+\sqrt{i \omega t}\right)+e^{-z \sqrt{i \omega}} \operatorname{Erfc}\left(\frac{z}{2 \sqrt{t}}-\sqrt{i \omega t}\right)\right)$

$f_{3}(z, t)=\frac{e^{-i \omega t}}{4}\left(e^{z \sqrt{-i \omega}} \operatorname{Erfc}\left(\frac{z}{2 \sqrt{t}}+\sqrt{-i \omega t}\right)+e^{-z \sqrt{-i \omega}} \operatorname{Erfc}\left(\frac{z}{2 \sqrt{t}}-\sqrt{-i \omega t}\right)\right)$

$f_{4}(z, t)=\operatorname{Erfc}\left(\frac{z}{2 \sqrt{t}}\right)$

Here, $\mathrm{z}$ is a dummy variable where $\mathrm{f} 1, \mathrm{f} 2, \mathrm{f} 3, \mathrm{f} 4$ are functions of dummy variable and $\operatorname{Erfc}(z)=$ $\frac{1}{2} \int_{0}^{\pi} e^{-t^{2}} \partial t$ is the complementary error function. Here, the subscripts $\mathrm{c}$ and $\mathrm{s}$ in the above equations refer to cosine and sine oscillations of the plate. On the other hand, the expression of Sherwood number, Nusselt number and skin friction for nanofluid flow is given as

$$
\begin{aligned}
& S h=\left.D_{n f} \frac{\partial C}{\partial t}\right|_{y=0} \\
& N u=-\left.\frac{k_{n f}}{k_{f}} \frac{\partial \theta}{\partial y}\right|_{y=0} \\
& \tau=-\left.\frac{1}{(1-\phi)^{2.5}} \frac{\partial u}{\partial y}\right|_{y=0}
\end{aligned}
$$




\section{Results and Discussions}

In this section, the graph of velocity, temperature and concentration profiles of nanofluid was plotted from the obtained exact solutions with different embedded parameters. Skin friction, Nusselt number and Sherwood number was also plotted from the obtained exact solutions using expression in Eq. (20) and Eq. (21). The graph was plotted using MATHEMATICA software. The result presented is from cosine oscillations solutions.

Figure 2 shows the concentration profiles graph with different Schmidt (Sc) number. Since Sc relates with relative thickness of the mass transfer boundary layer, increasing the Sc number will decrease the concentration. Figure 3 and Figure 4 show the temperature profile against Prandtl (Pr) number and nanoparticle volume fraction $(\phi)$. It shows that increasing Pr parameter will decreased the temperature of the nanofluid flow. Physically, the high number of Pr caused the thermal conductivity of fluid to decrease and the viscosity of the nanofluid increased resulting the temperature to decrease. While for $\phi$, increasing the nanoparticle volume fraction will cause the temperature to increase. It is proven that by adding a small number of nanoparticles on the fluid, will caused the thermal conductivity of the fluid to increased.

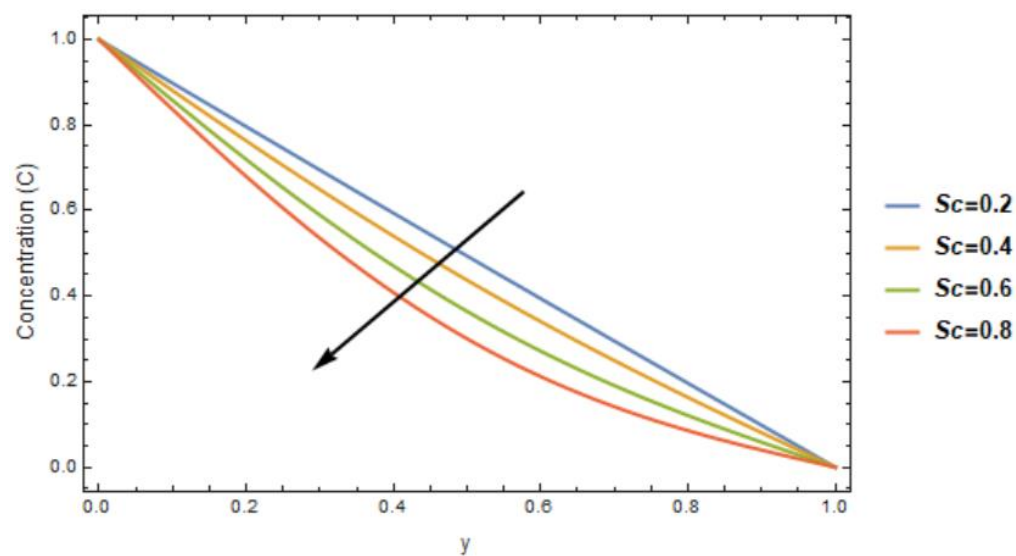

Fig. 2. Concentration profile with different Sc with $\phi=0.06, t=0.1$

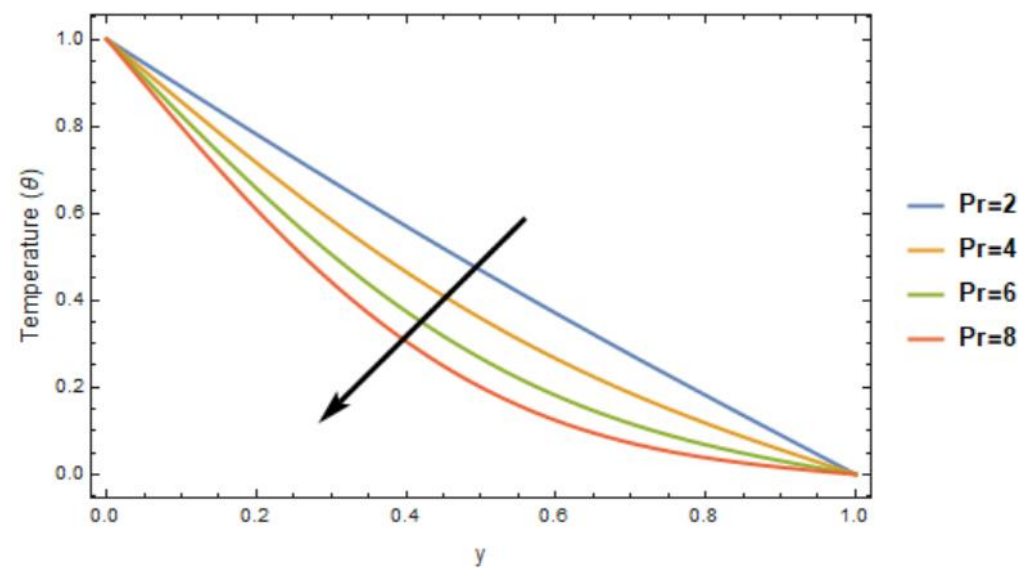

Fig. 3. Temperature profile with different $\operatorname{Pr}$ with $\phi=0.06, \mathrm{t}=0.1$ 


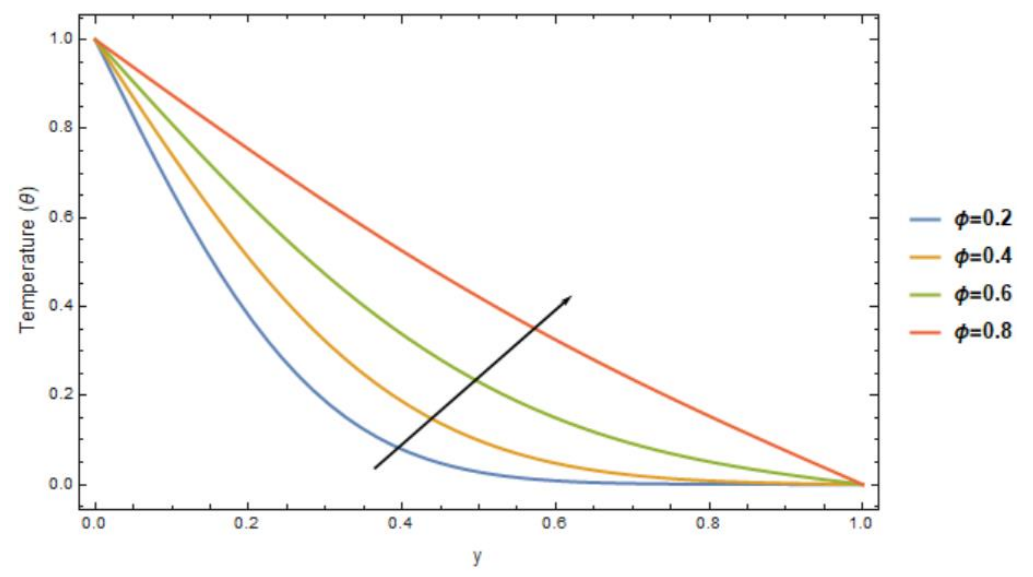

Fig. 4. Temperature profile with different $\phi$ with $\operatorname{Pr}=7, t=0.1$

Figure 5 to Figure 10 show the effects of different parameters Sc, Pr, Gr, Gm and $\phi$ on the velocity profile. From Figure 5, it was observed that increasing the Sc will decrease the velocity. It was found that Sc was relates to the relative thickness of the mass transfer boundary layer. If the Sc number is increases, the thickness of boundary layer would increase and will cause the velocity to decrease. While Figure 6 shows the velocity profile with different Pr. Increasing Pr will cause the viscosity to increase and will make the velocity to decrease. Figure 7 and Figure 8 show the velocity profiles with different Grashof number ( $\mathrm{Gr}$ ) and mass Grashof number $(\mathrm{Gm})$. It is observed that velocity increasing with increasing the value of Gr. Similar to Grashof number, when Gm increased, the velocity was found to increase. Physically, this is possible because as the Gr and Gm number increases, the buoyancy force near the plate causing a rise in velocity is observed. Figure 9 shows the velocity profile for different oscillation parameter. The graph shows that increasing the oscillation parameter will cause the velocity to decrease. Figure 10 shows velocity profile with different nanoparticles volume fraction. Increasing the nanoparticles volume fraction will decrease the velocity. A higher volume fraction of nanoparticles increases the nanofluid viscosity, which causes the fluid velocity to slow down.

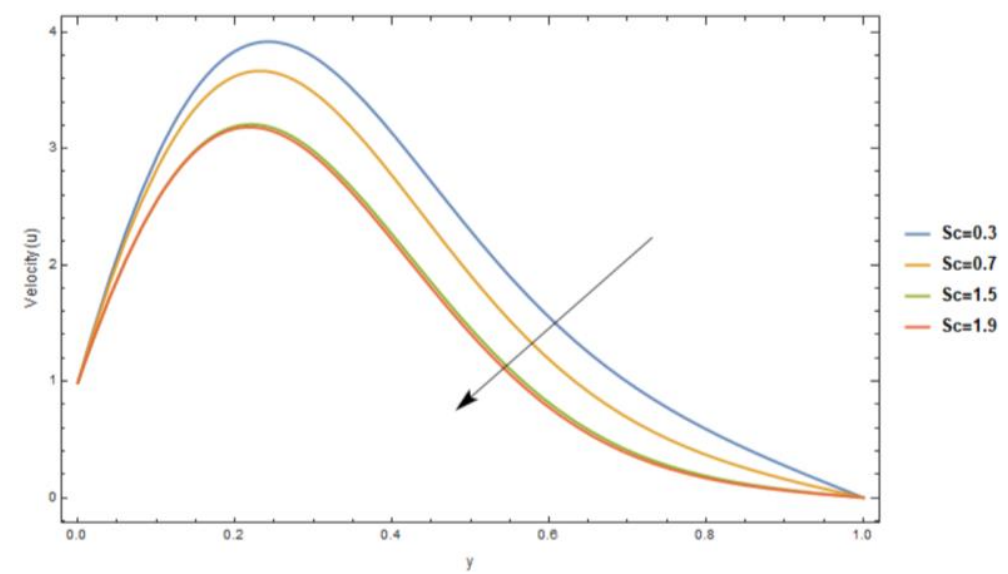

Fig. 5. Velocity profile with different Sc with $\operatorname{Pr}=7, \omega=\frac{\pi}{2}$, $\mathrm{Gr}=0.02, \mathrm{Gm}=0.02 \phi=0.06, \mathrm{t}=0.1$ 


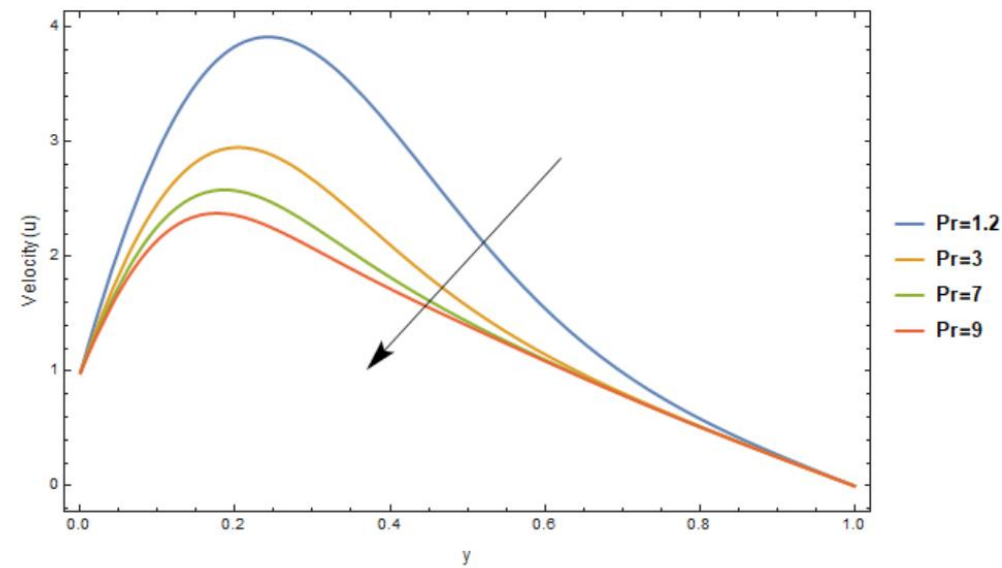

Fig. 6. Velocity profile with different $\operatorname{Pr}$ with $\mathrm{Sc}=0.5, \omega=\frac{\pi}{2}$, $\mathrm{Gr}=0.02, \mathrm{Gm}=0.02 \phi=0.06, \mathrm{t}=0.1$

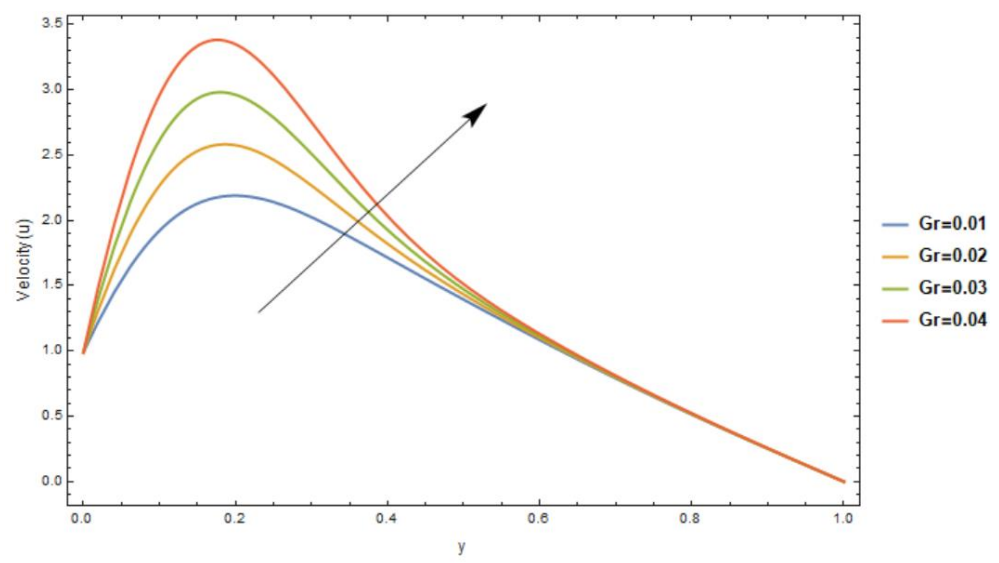

Fig. 7. Velocity profile with different $\mathrm{Gr}$ with $\operatorname{Pr}=7, \mathrm{Sc}=0.5, \omega=\frac{\pi}{2}$, $\mathrm{Gm}=0.02, \phi=0.06, \mathrm{t}=0.1$

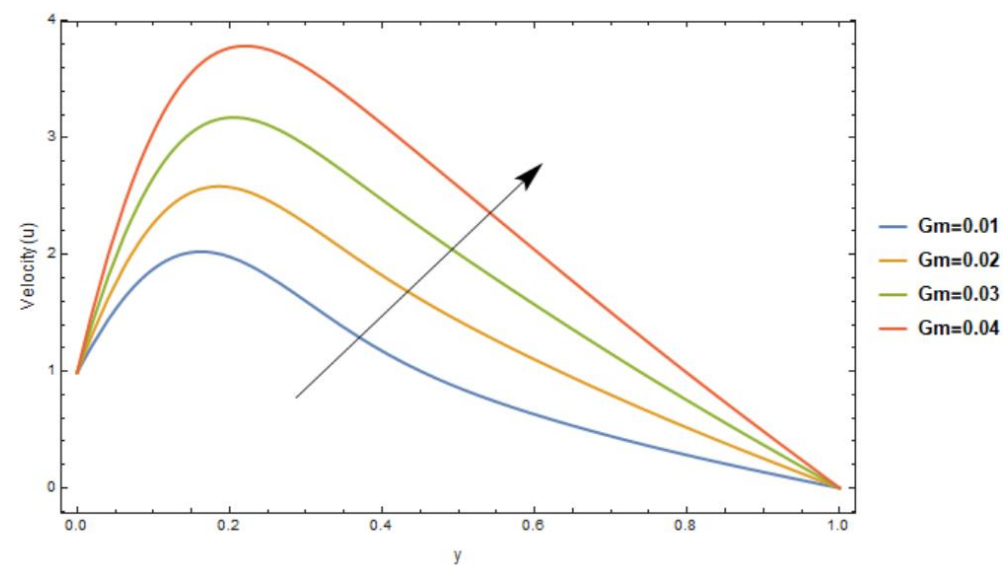

Fig. 8. Velocity profile with different $\mathrm{Gm}$ with $\mathrm{Pr}=7, \mathrm{Sc}=0.5$, $\omega=\frac{\pi}{2}, \mathrm{Gr}=0.02, \phi=0.06, \mathrm{t}=0.1$ 


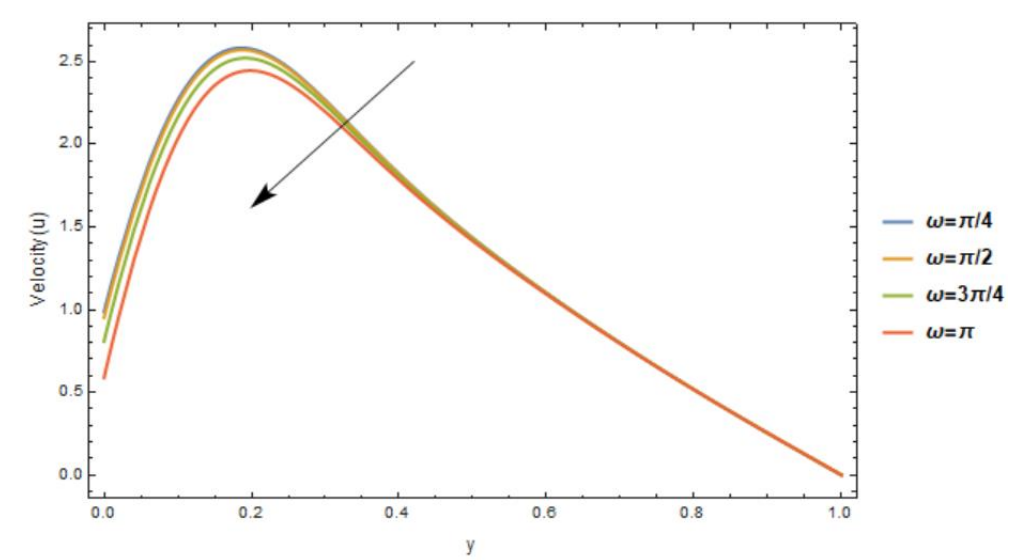

Fig. 9. Velocity profile with different $\omega$ with $\operatorname{Pr}=7, \mathrm{Sc}=0.5$, $\mathrm{Gm}=0.02, \mathrm{Gr}=0.02, \phi=0.06, \mathrm{t}=0.1$

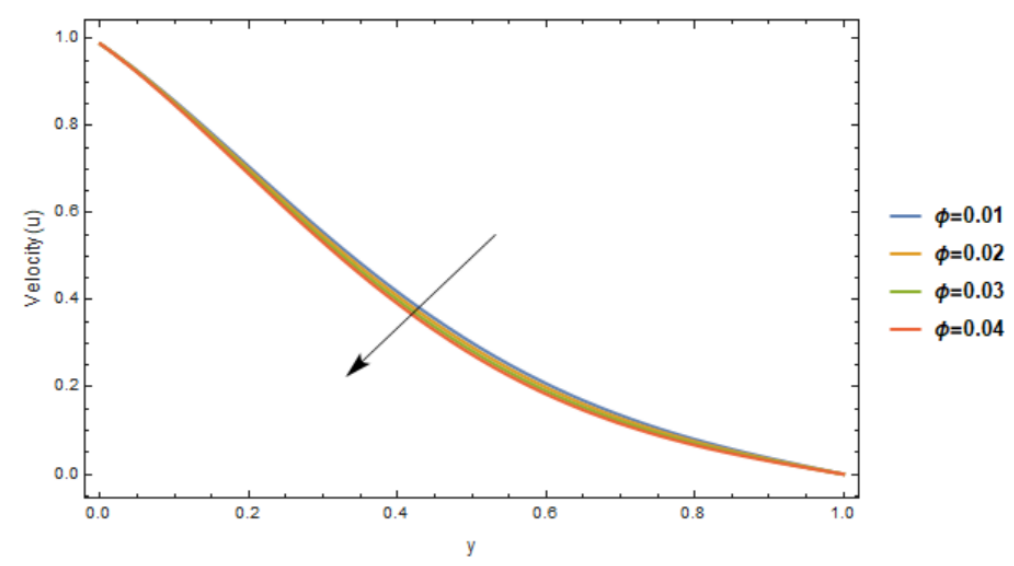

Fig. 10. Velocity profile with different $\phi$ with $\operatorname{Pr}=7, \mathrm{Sc}=0.5$, $\mathrm{Gm}=2, \mathrm{Gr}=2, \omega=\frac{\pi}{2}, \mathrm{t}=0.1$

Figure 11 and Figure 12 show skin friction graph for different $\operatorname{Pr}$ and $\phi$. It is depicted from the graph that increasing the Pr parameter will cause the skin friction to decrease but for $\phi$, skin friction increases with increasing $\phi$. The Nusselt number graph was plotted with different $\operatorname{Pr}$ and $\phi$ in Figure 13 and Figure 14. At a boundary in a fluid, the Nusselt number is the ratio of convective to conductive heat transfer. The conduction and convection heat flows are parallel to each other and to the surface normal of the boundary surface, and are all perpendicular to the mean fluid flow. By increasing both $\operatorname{Pr}$ and $\phi$ will cause the Nusselt number to increase. Figure 15 and Figure 16 show the graph of Sherwood number against time with different Sc number and $\phi$. Sherwood number represents the ratio between mass transfer by convection and mass transfer by diffusion. The graph shows that increasing the Sc number and $\phi$ will increase the Sherwood number. It is worth mentioning that all presented graphs show that the solutions satisfy the boundary conditions of the problem. Furthermore, the obtained solutions of concentration, temperature and velocity for cosine and sine fully satisfies the Eq. (8)-(10). This verification shows the correctness of the obtained solutions. 


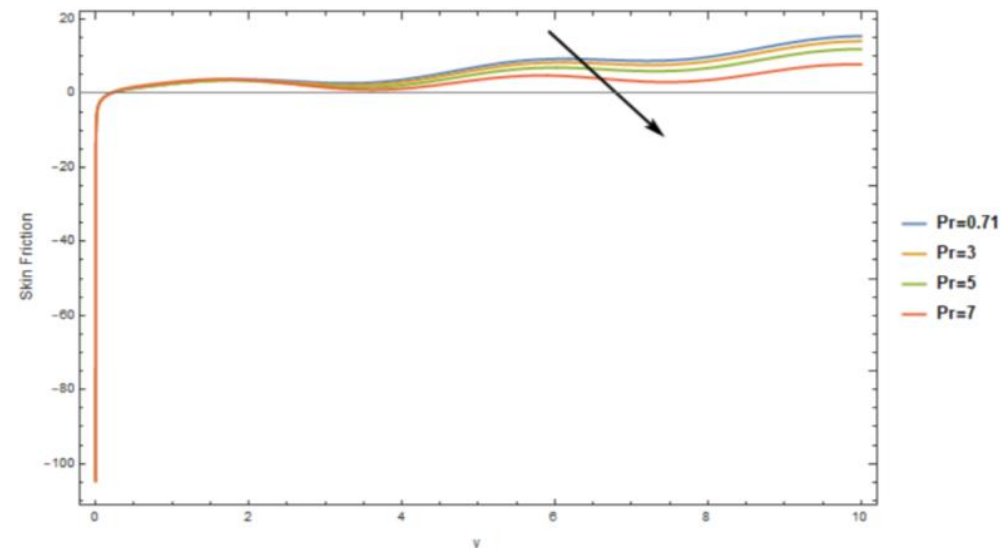

Fig. 11. Skin friction with different $\operatorname{Pr}$ with $\phi=0.06$, $S c=0.6$, $\mathrm{Gm}=2, \mathrm{Gr}=2, \omega=\frac{\pi}{2}$

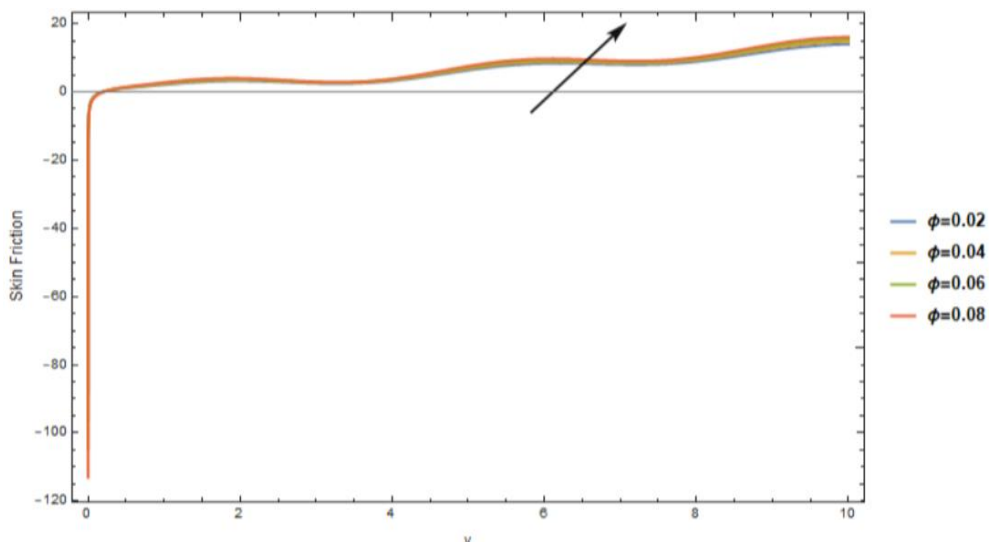

Fig. 12. Skin friction with different $\phi$ with $\operatorname{Pr}=0.2, \mathrm{Sc}=0.6, \mathrm{Gm}=2$, $\mathrm{Gr}=2, \omega=\frac{\pi}{2}$

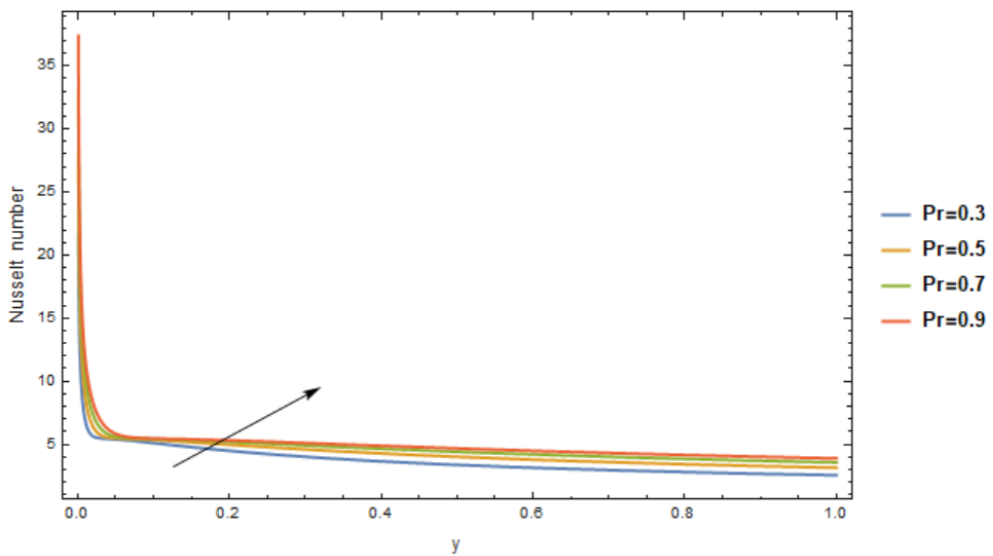

Fig. 13. Nusselt number with different $\operatorname{Pr}$ with $\phi=0.6$ 


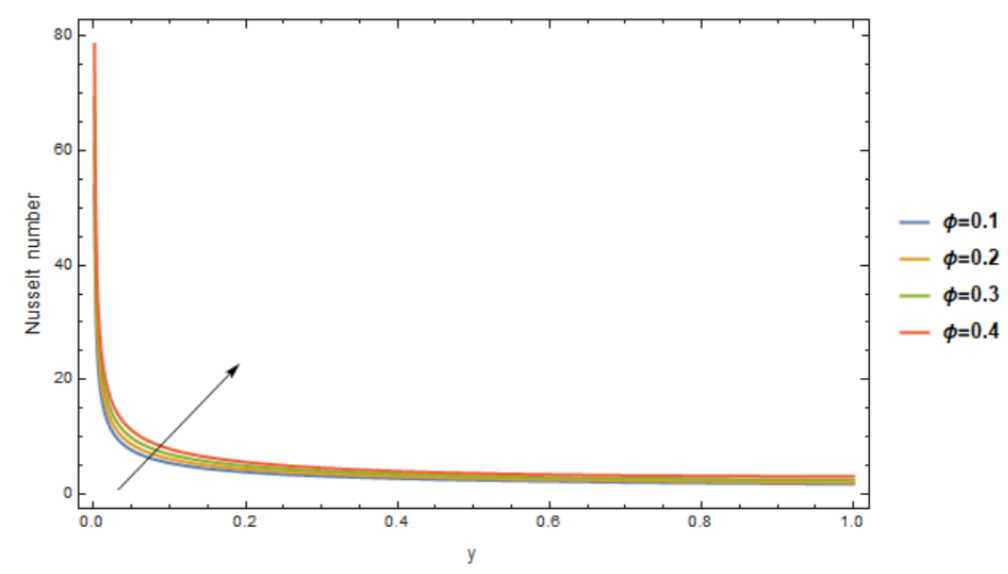

Fig. 14. Nusselt number with different $\phi$ with $\operatorname{Pr}=7$

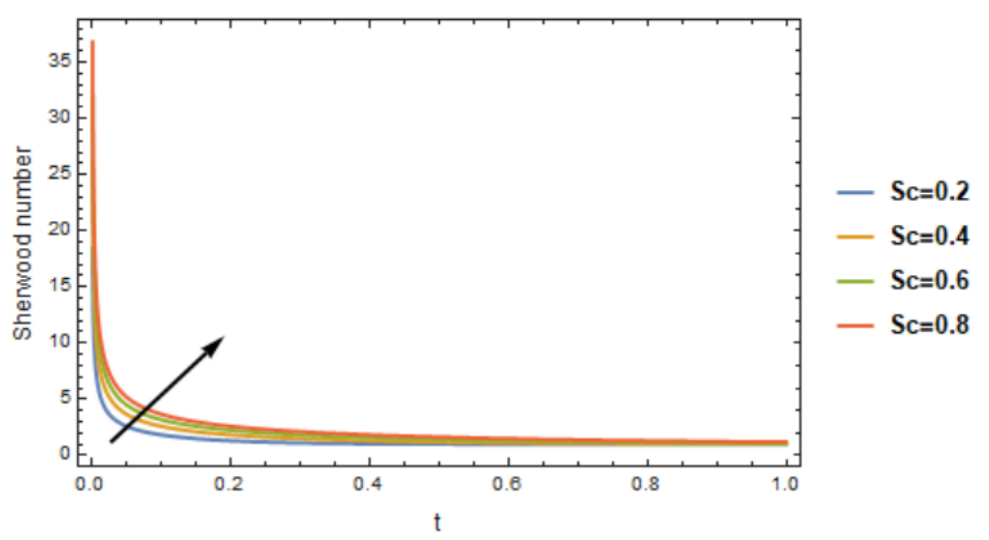

Fig. 15. Sherwood number with different Sc when $\phi=0.6$

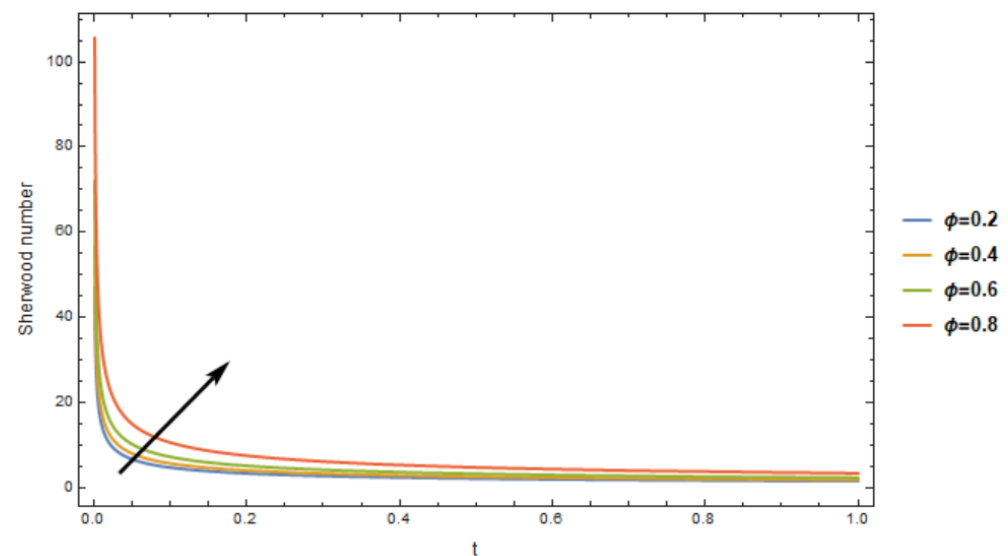

Fig. 16. Sherwood number with different $\phi$ with $\mathrm{Sc}=0.5$

\section{Conclusions}

Free convection nanofluid flow between oscillating vertical parallel plates with mass diffusion was studied. The nanofluid considered were $\mathrm{Cu}$-water. By using appropriate transformation, the equations were reduced to ordinary differential equations. Dimensionless governing equations were then solved using the Laplace transform method. Effects of various parameters on velocity, temperature and concentration profiles as well as Nusselt number, skin friction and Sherwood number were graphically studied in this paper. From this study, it was found that nanoparticles volume fraction $(\phi)$ plays a significant role in this research. Since $\phi$ will cause thermal conductivity 
of fluid to increase, the temperature, skin friction, Nusselt number and Sherwood number would increase when $\phi$ increases but will decrease in velocity profile. However, increasing the oscillation parameter will cause the velocity to decrease. Interestingly, the obtained solutions of velocity, temperature and concentration fully satisfied the dimensionless equations. The flow characteristics observed in this paper were found to be similar with Narahari et al., [16] which investigated exact solutions of free convection flow of nanofluids between two vertical parallel plates with radiation effect. Also, it is interesting to mention that exact solutions of velocity of Eq. (16), Eq. (17), temperature in Eq. (18) and concentration in Eq. (19) fully satisfied the non-dimensional Eq. (8) to Eq. (10). These ensure that all the obtained solutions are correct.

\section{Acknowledgement}

The authors would like to acknowledge the Ministry of Education (MOE), UTM Zamalah and Research Management Centre-UTM, Universiti Teknologi Malaysia (UTM) for the financial support through vote numbers 5F004, 5F116, 07G70, 07G72, 07G76, 07G77 and 17J98 for this research.

\section{References}

[1] Singh, A. K., Hamid R. Gholami, and V. M. Soundalgekar. "Transient free convection flow between two vertical parallel plates." Heat and Mass Transfer 31, no. 5 (1996): 329-331. https://doi.org/10.1007/s002310050063

[2] Marneni, Narahari. "Effects of thermal radiation and free convection currents on the unsteady Couette flow between two vertical parallel plates with constant heat flux at one boundary." WSEAS Transactions on Heat and Mass Transfer Issue (2010): 21-30.

[3] Rajput, U. S., and P. K. Sahu. "Transient free convection MHD flow between two long vertical parallel plates with constant temperature and variable mass diffusion." Journal of Math. Analysis 34, no. 5 (2011): 1665-1671.

[4] Vidhya, M., and Sundarammal Kesavan. "Laminar convection through porous medium between two vertical parallel plates with heat source." In Frontiers in Automobile and Mechanical Engineering-2010, pp. 197-200. IEEE, 2010. https://doi.org/10.1109/FAME.2010.5714846

[5] Umavathi, J. C., and M. Shekar. "Combined effect of variable viscosity and thermal conductivity on free convection flow of a viscous fluid in a vertical channel using DTM." Meccanica 51, no. 1 (2016): 71-86.

https://doi.org/10.1007/s11012-015-0202-4

[6] Falade, J. A., Joel C. Ukaegbu, A. C. Egere, and Samuel O. Adesanya. "MHD oscillatory flow through a porous channel saturated with porous medium." Alexandria Engineering Journal 56, no. 1 (2017): 147-152. https://doi.org/10.1016/i.aej.2016.09.016

[7] Hajizadeh, Ahmad, Nehad Ali Shah, Syed Inayat Ali Shah, I. L. Animasaun, Mohammad Rahimi-Gorji, and Ibrahim M. Alarifi. "Free convection flow of nanofluids between two vertical plates with damped thermal flux." Journal of Molecular Liquids 289 (2019): 110964.

https://doi.org/10.1016/j.molliq.2019.110964

[8] Hussanan, Abid, Muhammad Imran Anwar, Farhad Ali, Ilyas Khan, and Sharidan Shafie. "Natural convection flow past an oscillating plate with Newtonian heating." Heat Transfer Research 45, no. 2 (2014): 119-137. https://doi.org/10.1615/HeatTransRes.2013006385

[9] Chandrakala, P. "Radiation effects on flow past an impulsively started vertical oscillating plate with uniform heat flux." International Journal of Dynamics of Fluids 7, no. 1 (2011): 1-8.

[10] Chen, Yanyan, Ercang Luo, and Wei Dai. "Heat transfer characteristics of oscillating flow regenerator filled with circular tubes or parallel plates." Cryogenics 47, no. 1 (2007): 40-48.

https://doi.org/10.1016/i.cryogenics.2006.09.005

[11] Umavathi, J. C., A. J. Chamkha, A. Mateen, and A. Al-Mudhaf. "Unsteady Oscillatory Flow and Heat Transfer in a Horizontal Composite Porous Medium Channel." Nonlinear Analysis 14, no. 3 (2009): 397-415. https://doi.org/10.15388/NA.2009.14.3.14503

[12] Sharma, Pawan Kumar, and Mukesh Dutt. "MHD oscillatory free convection flow past parallel plates with periodic temperature and concentration." Universal Journal of Applied Mathematics 2, no. 7 (2014): 264-75.

[13] Das, S., Rabindra N. Jana, and Oluwole Daniel Makinde. "An oscillatory MHD convective flow in a vertical channel filled with porous medium with Hall and thermal radiation effects." Special Topics \& Reviews in Porous Media: An International Journal 5, no. 1 (2014). 
https://doi.org/10.1615/SpecialTopicsRevPorousMedia.v5.i1.60

[14] Choi, S. U. S., Z. G. Zhang, WLockwoodFE Yu, F. E. Lockwood, and E. A. Grulke. "Anomalous thermal conductivity enhancement in nanotube suspensions." Applied Physics Letters 79, no. 14 (2001): 2252-2254.

https://doi.org/10.1063/1.1408272

[15] Wong, Kaufui V., and Omar De Leon. "Applications of nanofluids: current and future." In Nanotechnology and Energy, pp. 105-132. Jenny Stanford Publishing, 2017. https://doi.org/10.1201/9781315163574-6

[16] Narahari, Marneni, Nikhila Alaparthi, and loan Pop. "Exact analysis of the transient free convection flow of nanofluids between two vertical parallel plates in the presence of radiation." The Canadian Journal of Chemical Engineering 95, no. 11 (2017): 2186-2198.

https://doi.org/10.1002/cjce.22872

[17] Hajizadeh, Ahmad, Nehad Ali Shah, Syed Inayat Ali Shah, I. L. Animasaun, Mohammad Rahimi-Gorji, and Ibrahim M. Alarifi. "Free convection flow of nanofluids between two vertical plates with damped thermal flux." Journal of Molecular Liquids 289 (2019): 110964.

https://doi.org/10.1016/j.molliq.2019.110964

[18] Sheikholeslami, M., and D. D. Ganji. "Heat transfer of Cu-water nanofluid flow between parallel plates." Powder Technology 235 (2013): 873-879.

https://doi.org/10.1016/j.powtec.2012.11.030

[19] Sheikholeslami, M., M. Hatami, and G. Domairry. "Numerical simulation of two phase unsteady nanofluid flow and heat transfer between parallel plates in presence of time dependent magnetic field." Journal of the Taiwan Institute of Chemical Engineers 46 (2015): 43-50.

https://doi.org/10.1016/i.jtice.2014.09.025

[20] Dogonchi, A. S., K. Divsalar, and D. D. Ganji. "Flow and heat transfer of MHD nanofluid between parallel plates in the presence of thermal radiation." Computer Methods in Applied Mechanics and Engineering 310 (2016): 58-76. https://doi.org/10.1016/i.cma.2016.07.003

[21] Pal, Dulal, Gopinath Mandal, and Kuppalapalle Vajravalu. "Soret and Dufour effects on MHD convective-radiative heat and mass transfer of nanofluids over a vertical non-linear stretching/shrinking sheet." Applied Mathematics and Computation 287 (2016): 184-200.

https://doi.org/10.1016/i.amc.2016.04.037

[22] Feng, Xuemei, and Drew W. Johnson. "Mass transfer in SiO2 nanofluids: a case against purported nanoparticle convection effects." International Journal of Heat and Mass Transfer 55, no. 13-14 (2012): 3447-3453.

https://doi.org/10.1016/i.ijheatmasstransfer.2012.03.009 